\title{
The role of Dexamethasone in clinical pharmaceutical treatment for patients with cataract surgery
}

\author{
GAOKUN ZHANG ${ }^{1}$, SHUANG LIU ${ }^{2}$, LIEYING YANG ${ }^{3}$ and YANQING LI $^{3}$ \\ ${ }^{1}$ Department of Nursing; ${ }^{2}$ Emergency Internal Medicine; ${ }^{3}$ Department of Ophthalmology, \\ Mudanjiang Medical University of Affiliated HongQi Hospital, Mudanjiang, Heilongjiang 157000, P.R. China
}

Received March 13, 2017; Accepted August 24, 2017

DOI: 10.3892/etm.2017.5639

\begin{abstract}
The aim of the present study is to determine the efficacy of dexamethasone (DEX) vs. indomethacin (IND) and ciprofloxacin (CIP) in modulating immediate inflammation following cataract surgery. A total of 644 patients with cataract were recruited and inflammation was investigated during the perioperative period. The management protocol was similar in both groups. Each drug was given four times a day for 30 days starting 7 days prior to surgery. The primary efficacy criteria for evaluation were the reduction in anterior chamber (AC) flare and AC inflammation score, in addition, a different secondary efficacy and safety evaluation criteria were maintained. The results demonstrated that the average inflammation score was $1.2 \pm 0.8$ in the DEX group, which

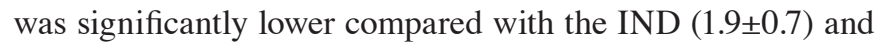
CIP (1.8 \pm 0.6$)$ groups. Intra-ocular pressure was decreased following treatment with DEX, while intra-ocular pressure was slightly increased in the IND $(1.9 \pm 0.7)$ and CIP $(1.8 \pm 0.6)$ groups. The final visual outcomes for patients postcataract surgery were clinically superior in the DEX group compared with that of the IND and CIP groups $(\mathrm{P}=0.034$ and $\mathrm{P}=0.042$, respectively), but there was no significant difference between the ND and CIP groups ( $\mathrm{P}=0.78)$. Outcomes indicated that $\mathrm{AC}$ inflammation, conjunctival hyperaemia, corneal and lid oedema, ocular infection, pain, photophobia, and tearing were significantly improved in each group. No significant poor local tolerance or adverse reaction was observed in the DEX, IND and CIP groups. In conclusion, the outcomes of the present study suggest that DEX serves an important role in the clinical pharmaceutical treatment of patients with cataract surgery.
\end{abstract}

Correspondence to: Dr Yanqing Li, Department of Ophthalmology, Mudanjiang Medical University of Affiliated HongQi Hospital, 2 Taiping Road, Mudanjiang, Heilongjiang 157000, P.R. China E-mail: liyanqingdoc@sohu.com

Key words: cataract, dexamethasone, clinical pharmaceutical treatment, anterior chamber inflammation

\section{Introduction}

Cataract is one of the most common eye diseases that caused by various factors and leads to the lens protein denaturation and cloudy (1). Cataract includes congenital cataract and acquired cataract. Clinical survey presents that cataract can be induced by aging, genetic, local nutritional disorders, immune and metabolic abnormalities, trauma, poisoning, and radiation (2). Currently, drug treatments and surgery are two common clinical projects of treatment for cataract patients. Cataract phacoemulsification has widely used for cataract patients due to small incision, less tissue injury, short operation time and visual acuity (3). Clinical pharmaceutical nursing is essential for patients after surgery for patients with cataract (4).

Dexamethasone (DEX) is an effective drug in clinical pharmaceutical treatment for patients with cataract surgery (5). Missotten et al have compared the efficacy and safety of topical $0.1 \%$ indomethacin with $0.1 \%$ DEX after cataract surgery and results identified that $0.1 \%$ dexamethasone eyedrops manage is safe and efficient in the management of post-operative inflammation and could be a good alternative to the use of steroids (6). Notably, the efficacy and safety of DEX-netilmicin has analyzed in patients after cataract surgery (7). However, the systematic study of DEX in reduction of AC inflammation, improvement of visual acuity local tolerance or adverse reaction has not well understood yet.

Previous reports have indicated that DEX, indomethacin (IND), ciprofloxacin (CIP) presented anti-inflammation activity for patients with cataract (8-11). On these premises, we sought to compare anti-inflammation among DEX, IND and CIP in controlling immediate inflammation after cataract surgery in clinical treatment. This prospective, multi-center, clinical parallel group study assesses the efficacy and safety of DEX for post-cataract inflammation, intra-ocular pressure and final visual outcomes.

\section{Materials and methods}

Study design, subjects and sampling. A total of 644 Han Chinese People cataract patients were recruited in this analysis. The age of patient's was 50.2-64.6 years old. The characteristics of patients with cataract were summarized in Table I. Inclusion criteria for individuals with cataract were diagnosed slit-lamp examination, measurement of best corrected visual acuity 
(BCVA), intra-ocular pressure, fundoscopy, and a complete medical history. The Institutional Review Board approval was obtained for this study. The study protocol was approved by the Central Ethics Committee (Ethics Committee of Hongqi Hospital of Mudanjiang Medical College). All patients were required to write informed consent with signature.

Drugs administration. In total, 644 patients were enrolled in this study and were randomized into three groups. All of the patients completed both the study and the follow-up period. The indicated dosage of ophthalmic solutions was DEX $0.1 \%$ (Tianjin day medicine pharmaceutical co., LTD), IND $0.1 \%$ (Shanghai Xinyi Jiufu pharmaceutical co., LTD) and CIP $0.1 \%$ (Wuhan wujing pharmaceutical co., LTD) administered at one drop in the treated eye ten times every day. Patients with cataract were given DEX $0.1 \%$, IND $0.1 \%$ and CIP $0.1 \%$ prior phacoemulsification 7 days and post phacoemulsification surgery 23 days.

Surgical technique. All patients with cataract were undergone a continuous curvilinear phacoemulsification using a small incision with the Bausch \& Lomb Stellaris system. An acrylic foldable IOL was then implanted in the capsular bag in cataract patients. All operations were performed by one surgeon descripted previously (12). The corneal thickness was measured using an Orbscan II (Bausch \& Lomb, Rochester, NY, USA) and effective phacoemulsification times in three groups were recorded.

AC inflammation severity score and chamber flare. Criteria for evaluation were the reduction in anterior chamber flare and anterior chamber (AC) inflammation severity score (primary efficacy criteria) as well as different secondary efficacy and safety evaluation criteria. Anterior chamber flare was detected by using the Kowa FM-600 laser flare meter (Hamamatsu PM; Hamamatsu Photonics, Hamamatsu City, Japan). Inflammation severity in patients were examined with a laser cell flare meter FC-2000 (Kowa Company, Ltd., Electronics and Optics Division, Tokjo, Japan) which quantifies aqueous humor proteins and cells. Clinical assessment of intraocular inflammation: The laser cell flare meter which determines protein concentration and cell number in aqueous humor in vivo (13). Mean AC inflammation severity score were evaluated according to previous report (14). A criterion for evaluation for patients was measurement of visual acuity, the reduction in anterior chamber flare and inflammation severity score.

Intraocular pressure measurement. Corneal surface intraocular pressure in each patients prior and post phacoemulsification surgery was measured using a Tono-Pen AVIA ${ }^{\circledR}$ Applanation Tonometer (Reichert Technologies, USA). To minimize circadian oscillation, intraocular pressure measurement measurements were performed at 12:30 pm in all patients.

Clinical assessments. All measurements were performed by the same technician in three groups during the preoperative period, on day 7 prior phacoemulsification surgery (day 1), and on day 23 post phacoemulsification surgery. The efficacy of DEX, IND and CIP on anterior AC inflammation, conjunctival hyperaemia, corneal and lid oedema, ocular infection, pain, photophobia and tearing were recorded using person's own records and analyzed using scoring systems for the signs and symptoms. Each result of aqueous flare or corneal thickness was determined based on the mean of five measurements.

Statistical analysis. Continuous variables were shown as mean \pm SD and analyzed by students t test. All data were analyzed using SPSS Statistics 19.0 (version 19.0; SPSS Inc., Chicago, IL, USA) and Graphpad Prism version 5.0 with the help of Microsoft Excel. Unpaired data was determined by Student's t test and comparisons of data between multiple groups were analyzed by variance (ANOVA). A P-value of $\leq 0.05$ was considered statistically significant.

\section{Results}

The efficacy of DEX on inflammation in patients post cataract surgeries during perioperative period. All the cataract patients completed follow-up period. Patients were undergone effective phacoemulsification surgery. The effective phacoemulsification times in three groups were similar and no significant differences were found between the three groups in corneal thickness at the three time points $(\mathrm{P}>0.05$ for postoperative group; Fig. 1). There were no significant differences between the three groups in corneal thickness and phacoemulsification surgery time among patients (Table II). Outcomes demonstrated that average AC inflammation score was lower in DEX group (1.2 \pm 0.8$)$ than in IND $(1.9 \pm 0.7)$ and CIP $(1.8 \pm 0.6)$ groups $(\mathrm{P}<0.05$, Fig. 2). Aqueous flare examination also showed that DEX, IND and CIP significantly improved aqueous for patients after phacoemulsification surgery (Fig. 3). These results indicate that DEX can decrease AC inflammation for patients post cataract surgery during perioperative period.

The efficacy of DEX on intra-ocular pressure in patients post cataract surgeries during perioperative period. Intra-ocular pressure was measured in patients with cataract prior and post cataract surgery in DEX, IND and CIP groups. We showed that DEX treatment decreased intra-ocular pressure compared to IND and CIP groups, while no significant difference between IND and CIP group during perioperative period (Fig. 4). These outcomes suggest that DEX is more efficient in improving intra-ocular pressure than IND and CIP.

The efficacy of DEX on final visual outcomes in patients post cataract surgeries during perioperative period. We analyzed the efficacy of DEX on final visual outcomes in patients post cataract surgeries during perioperative period. As shown in Fig. 5, DEX treatment significantly improved final visual outcomes compared to IND and CIP groups. Results demonstrated mean best-corrected visual acuity significantly improved from 0.38 at baseline to 0.11 in DEX group. However, mean best-corrected visual acuity was improved from 0.39 , 0.38 at baseline to 0.15 and 0.16 in IND and CIP group, respectively. More importantly, the completed degree showed that DEX treatment significantly improved retinoschisis compared to IND and CIP groups (Fig. 6). These results indicate that DEX treatment is beneficial for final visual improvements in patients post cataract surgeries during perioperative period. 
Table I. The clinical characteristics of cataract patients and healthy individuals.

\begin{tabular}{lccc}
\hline Characteristic & Male & Female & P-value \\
\hline Age-years & $50.2-62.8$ & $52.4-64.6$ & $>0.05$ \\
Numbers & 318 & 326 & $>0.05$ \\
Corneal thickness $(\mu \mathrm{m})$ & $532.3 \pm 46.4$ & $542.2 \pm 78.2$ & $>0.05$ \\
Inflammation severity & $3.0 \pm 1.0$ & $2.8 \pm 1.2$ & $>0.05$ \\
Intraocular pressure (mm Hg) & $15.6 \pm 2.0$ & $16.2 \pm 1.8$ & $>0.05$ \\
Aqueous flare (p/msec) & $8.6 \pm 2.2$ & $9.2 \pm 3.0$ & $>0.05$ \\
Phacoemulsification surgery time(s) & $9.41 \pm 1.37$ & $9.57 \pm 1.39$ & $>0.05$ \\
DEX & 110 & 108 & $>0.05$ \\
IND & 106 & 112 & $>05$ \\
CIP & 97 & 91 & $>0.05$ \\
\hline
\end{tabular}

Table II. Corneal thickness and phacoemulsification surgery time for patients prior and post cataract surgery.

\begin{tabular}{lcrr}
\hline Parameters & DEX & IND & CIP \\
\hline Corneal thickness & & & \\
Prior cataract surgery & $548.64 \pm 54.88$ & $562.4 \pm 60.32$ & $555.28 \pm 56.82$ \\
Post cataract surgery & $536.64 \pm 42.38$ & $560.10 \pm 72.34$ & $560.60 \pm 58.94$ \\
Phacoemulsification surgery time(s) & $9.56 \pm 1.80$ & $9.64 \pm 1.90$ & $9.57 \pm 1.86$ \\
\hline
\end{tabular}

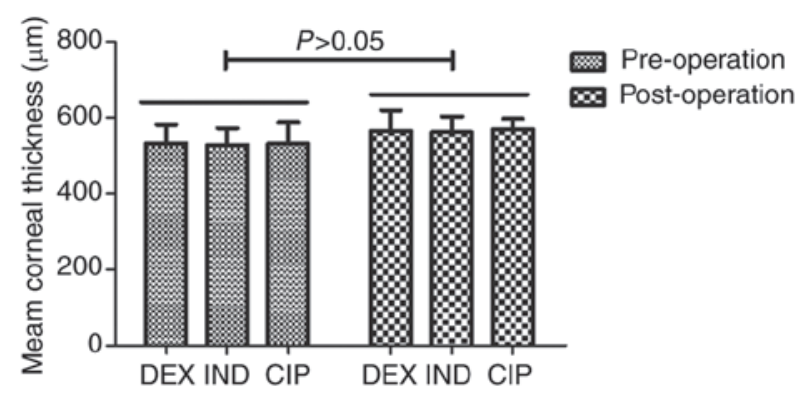

Figure 1. The effective phacoemulsification times for patients with cataract in DEX, IND and CIP groups. DEX, dexamethasone; IND, indomethacin; CIP, ciprofloxacin.

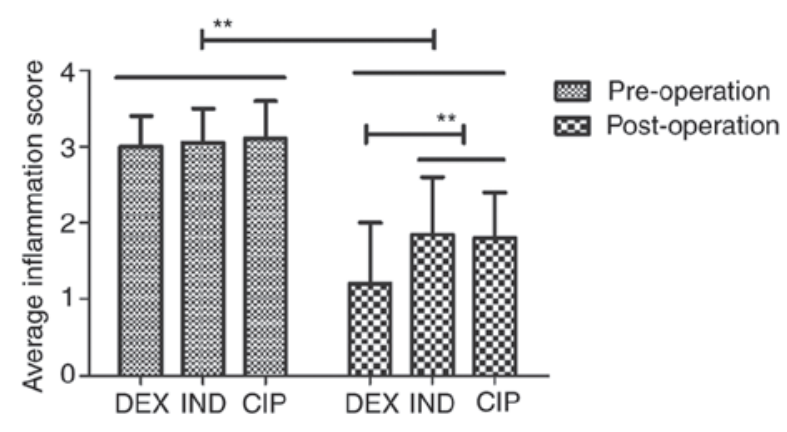

Figure 2. Average AC inflammation score for patients with cataract in DEX, IND and CIP groups prior and post phacoemulsification surgery. DEX, dexamethasone; IND, indomethacin; CIP, ciprofloxacin. ${ }^{* *} \mathrm{P}<0.01$.

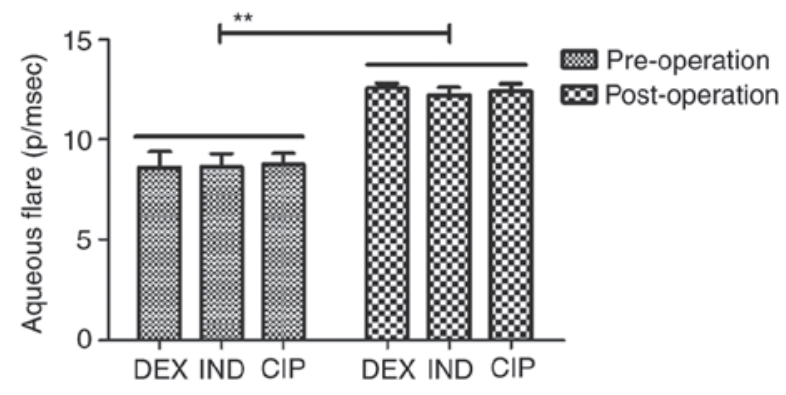

Figure 3. DEX, IND and CIP significantly improves aqueous for patients after phacoemulsification surgery. DEX, dexamethasone; IND, indomethacin; CIP, ciprofloxacin. ${ }^{* *} \mathrm{P}<0.01$.

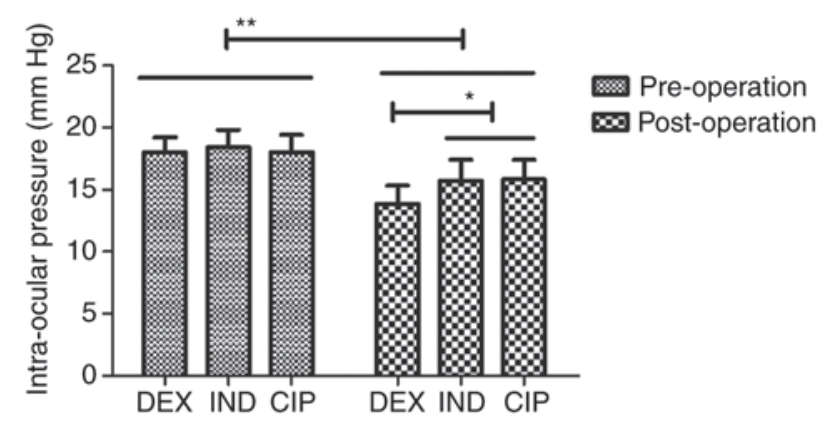

Figure 4. DEX, IND and CIP significantly decreases intra-ocular pressure for patients after phacoemulsification surgery. DEX, dexamethasone; IND, indomethacin; CIP, ciprofloxacin. ${ }^{*} \mathrm{P}<0.05,{ }^{* * *} \mathrm{P}<0.01$.

to analyze the efficacy of DEX for patients with cataract after phacoemulsification surgery. As shown in Fig. 7, ocular 


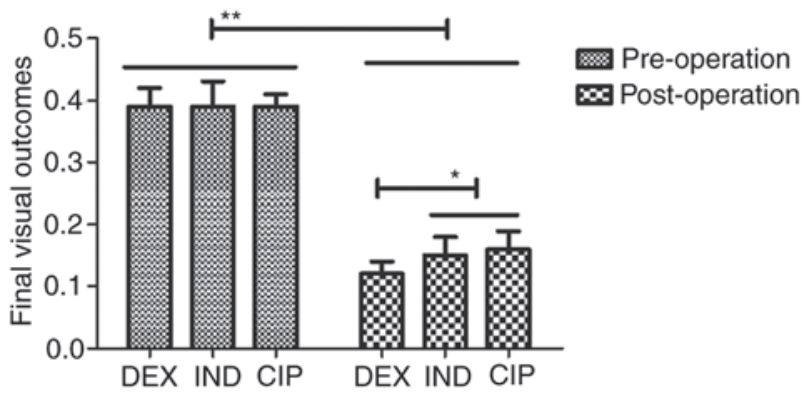

Figure 5. DEX significantly improves final visual outcomes compared to IND and CIP groups. DEX, dexamethasone; IND, indomethacin; CIP, ciprofloxacin. $\mathrm{P}<0.05,{ }^{* *} \mathrm{P}<0.01$.

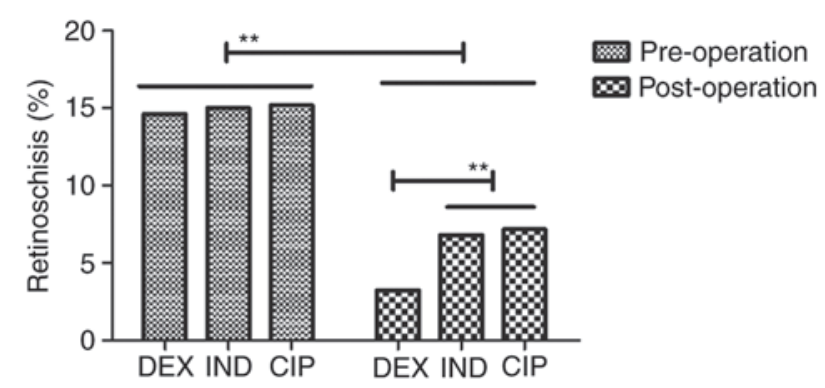

Figure 6. DEX significantly meliorated retinoschisis compared to IND and CIP groups. DEX, dexamethasone; IND, indomethacin; CIP, ciprofloxacin. ${ }^{* *} \mathrm{P}<0.01$.

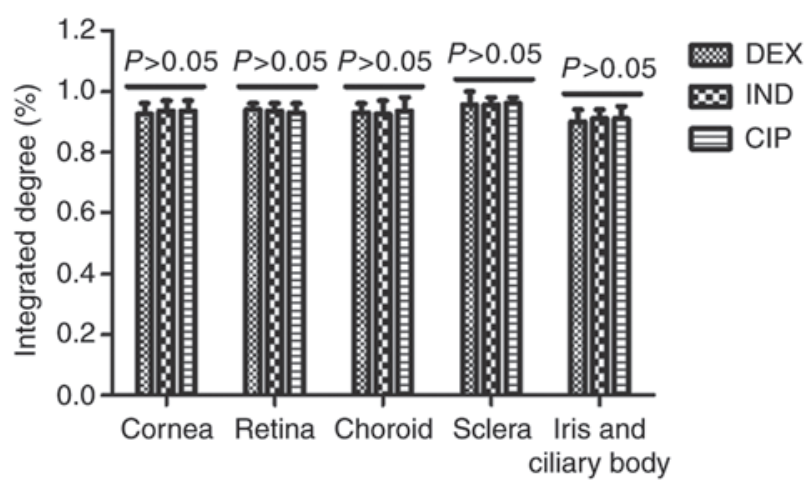

Figure 7. DEX, IND and CIP is safe agent for patients after phacoemulsification surgery. DEX, dexamethasone; IND, indomethacin; CIP, ciprofloxacin.

tissues-cornea, iris/ciliary body and retina/choroid/sclera showed normal tissues in all three groups. Outcomes indicated that $\mathrm{AC}$ inflammation, conjunctival hyperaemia, corneal and lid oedema, ocular infection, pain, photophobia and tearing were significantly improved in DEX, IND and CIP groups. These results suggest that DEX is an efficient and safe drug for patients post cataract surgeries during perioperative period.

\section{Discussion}

Cataract is one kind eye diseases, which is derived from metabolic disorders in crystalline lens $(15,16)$. Decreasing of AC inflammation contributes to final visual outcomes for patients with cataract after surgery $(11,17)$. Reports have indicated that the efficacy and safety of DEX for patients after cataract surgery and outcomes have showed DEX is potential drug for the prevention and treatment of retinal vein occlusion and non-infectious uveitis $(18,19)$. In addition, DEX management decreased inflammation for uveitis and postoperative cataract inflammation (20). In this study, we investigated the ameliorative effects of DEX in decreasing AC inflammation compared to IND and CIP groups. Findings in this study have showed the efficacy of DEX on AC inflammation, intra-ocular pressure, final visual outcomes and safety for cataract patients after phacoemulsification surgery. We showed that DEX presents more efficient for improvements of inflammation, intra-ocular pressure and final visual outcomes compared to IND and CIP groups. The efficacy of DEX, IND and CIP was also evaluated determined by $\mathrm{AC}$ inflammation, conjunctival hyperaemia, corneal and lid oedema, ocular infection, pain, photophobia and tearing in patients after cataract surgery.

Currently, comparative studies of anti-inflammatory drugs for control of post cataract surgery AC inflammation have been proposed in clinic (21-23). Previous study has evaluated the efficacy of intracameral dexamethasone predisposes to glaucoma after pediatric cataract surgery (24). In addition, clinical study has presented the tolerance and efficacy of piroxicam used in cataract surgery, compared with diclofenac and indomethacin (25). Weber et al (11) also indicated the efficacy and safety of IND eye drops compared with ketorolac $0.5 \%$ eye drops in the management of ocular inflammation after cataract surgery. Further, anti-inflammation of CIP effects for inhibition of conjunctival bacterial flora after cataract surgery has been analyzed in a controlled-release system (10). In this study, we compared the efficacy of DEX, IND and CIP in improving AC inflammation, intra-ocular pressure and final visual outcomes. Our outcomes indicate that DEX is more efficient in anti-AC inflammation, improving intra-ocular pressure and final visual outcomes.

Results showed that average inflammation score is $1.2 \pm 0.8$ in DEX group, while it was $(1.9 \pm 0.7)$ and $(1.8 \pm 0.6)$ in IND and CIP group, respectively. We found that DEX treatment significantly improved intra-ocular pressure and final visual outcomes compared to IND and CIP group for cataract patients after phacoemulsification surgery. However, the ameliorative efficacy for AC inflammation, conjunctival hyperaemia, corneal and lid oedema, ocular infection, pain, photophobia and tearing were confirmed and no significant difference among DEX, IND and CIP groups. These outcomes indicate that DEX provides insights into the importance of clinical treatment for AC inflammation and visual acuity in cataract patients after phacoemulsification surgery.

In conclusion, these short-term results suggest that a single DEX treatment is more efficient for cataract patients, which could avoid an increase in inflammation, central retinal thickness and intra-ocular pressure after cataract surgery. We will explore whether combination of DEX, IND and CIP will be generate synergetic effects to control postoperative inflammation and uveitis without regard to patient compliance and therefore enhance clinical outcomes.

\section{References}

1. Bilge AH, Aykan U, Akin T and Unsal U: Review of sterile, postoperative, anterior segment inflammation following cataract extraction and intraocular lens implantation. Eur J Ophthalmol 15: 224-227, 2005. 
2. Benemei S, Nicoletti P, Geppetti P and Bonciani M: Cataract in chronic cluster headache: Two case reports and review of the literature. J Headache Pain 9: 401-403, 2008.

3. Drolsum L, Ringvold A and Nicolaissen B: Cataract and glaucoma surgery in pseudoexfoliation syndrome: A review. Acta Ophthalmol Scand 85: 810-821, 2007.

4. Subzwari S, Desapriya E, Scime G, Babul S, Jivani K and Pike I: Effectiveness of cataract surgery in reducing driving-related difficulties: A systematic review and meta-analysis. Inj Prev 14: 324-328, 2008.

5. Laurell CG and Zetterström C: Effects of dexamethasone, diclofenac, or placebo on the inflammatory response after cataract surgery. Br J Ophthalmol 86: 1380-1384, 2002.

6. Missotten L, Richard C and Trinquand C: Topical $0.1 \%$ indomethacin solution versus topical $0.1 \%$ dexamethasone solution in the prevention of inflammation after cataract surgery. The study group. Ophthalmologica 215: 43-50, 2001.

7. Russo S, Papa V, Di Bella A, Favero A, Radulescu C, Gafencu O, Carstocea B and Milazzo G: Dexamethasone-netilmicin: A new ophthalmic steroid-antibiotic combination. Efficacy and safety after cataract surgery. Eye (Lond) 21: 58-64, 2007.

8. Carron A, Samudio M, Laspina F, Fariña N, Sanabria RR, Cibils D, Ramirez L, Carron J and Mino de Kaspar H: Efficacy of topical $0.3 \%$ ciprofloxacin application in reducing the conjunctival biota of patients undergoing cataract extraction. Arch Soc Esp Oftalmol 88: 345-351, 2013 (In Spanish).

9. Calvo P, Ferreras A, Al Adel F, Dangboon W and Brent MH: Effect of an intravitreal dexamethasone implant on diabetic macular edema after cataract surgery. Retina, Feb 10, 2017 (Epub ahead of print).

10. Paganelli F, Cardillo JA, Melo LA Jr, Lucena DR, Silva AA Jr, Oliveira AG, Höfling-Lima AL, Nguyen QD, Kuppermann BD and Belfort R Jr; Brazilian Ocular Pharmacology and Pharmaceutical Technology Research Group: A single intraoperative sub-tenon's capsule injection of triamcinolone and ciprofloxacin in a controlled-release system for cataract surgery. Invest Ophthalmol Vis Sci 50: 3041-3047, 2009.

11. Weber M, Kodjikian L, Kruse FE, Zagorski Z and Allaire CM: Efficacy and safety of indomethacin $0.1 \%$ eye drops compared with ketorolac $0.5 \%$ eye drops in the management of ocular inflammation after cataract surgery. Acta Ophthalmol 91: e15-e21, 2013.

12. Bhargava R, Kumar P, Sharma SK and Arora Y: Phacoemulsification versus manual small incision cataract surgery in patients with fuchs heterochromic iridocyclitis. Asia Pac J Ophthalmol (Phila) 5: 330-334, 2016.
13. Sawa M, Tsurimaki Y, Tsuru T and Shimizu H: New quantitative method to determine protein concentration and cell number in aqueous in vivo. Jpn J Ophthalmol 32: 132-142, 1988.

14. Lane SS and Holland EJ: Loteprednol etabonate $0.5 \%$ versus prednisolone acetate $1.0 \%$ for the treatment of inflammation after cataract surgery. J Cataract Refract Surg 39: 168-173, 2013.

15. Ozcan H, Yucel A and Ates O: Visual hallucinations in an old patient after cataract surgery and treatment. Eurasian J Med 48: 62-64, 2016.

16. Tsilimbaris MK, Tsika C and Kymionis GD: Intravitreal ketorolac for the treatment of chronic cystoid macular edema after cataract surgery. Ther Clin Risk Manag 12: 177-182, 2016.

17. Cho H, Wolf KJ and Wolf EJ: Management of ocular inflammation and pain following cataract surgery: Focus on bromfenac ophthalmic solution. Clin Ophthalmol 3: 199-210, 2009.

18. Meyer LM and Schönfeld CL: Cystoid macular edema after complicated cataract surgery resolved by an Intravitreal Dexamethasone 0.7-mg implant. Case Rep Ophthalmo 2: 319-322, 2011.

19. Pianini V, Passani A, Rossi GC and Passani F: Efficacy and safety of netilmycin/dexamethasone preservative-free and tobramycin/dexamethasone-preserved fixed combination in patients after cataract surgery. J Ocul Pharmacol Ther 26: 617-621, 2010.

20. Chennamaneni SR, Mamalis C, Archer B, Oakey Z and Ambati BK: Development of a novel bioerodible dexamethasone implant for uveitis and postoperative cataract inflammation. J Control Release 167: 53-59, 2013.

21. Malik A, Sadafale A, Gupta YK and Gupta A: A comparative study of various topical nonsteroidal anti-inflammatory drugs to steroid drops for control of post cataract surgery inflammation. Oman J Ophthalmol 9: 150-156, 2016.

22. Kaur S and Sukhija J: Difluprednate versus prednisolone acetate for inflammation following cataract surgery in pediatric patients: A randomized safety and efficacy study. Eye (Lond) 31: 506, 2017.

23. Wilson ME, Lambert SR, Plager DA, VanderVeen D, Roarty J and O'Halloran H: Difluprednate versus prednisolone acetate for inflammation following cataract surgery in pediatric patients: A randomized safety and efficacy study. Eye (Lond) 31: 506-507, 2017.

24. Mataftsi A, Dabbagh A, Moore W and Nischal KK: Evaluation of whether intracameral dexamethasone predisposes to glaucoma after pediatric cataract surgery. J Cataract Refract Surg 38: 1719-1723, 2012.

25. Costin D, Popa S and Costea C: Clinical study on the tolerance and efficacy of piroxicam used in cataract surgery, compared with diclofenac and indomethacin. Rev Med Chir Soc Med Nat Iasi 109: 305-313, 2005. 